\title{
Detection of Ultralow Light Power Back-Reflected or Back-Scattered by Optical Components Using Balanced Low-Coherence Interferometry
}

\author{
Imran Khan, Michel Lequime $\odot,{ }^{*}$ Myriam Zerrad, and Claude Amra \\ Aix Marseille Université, CNRS, Centrale Marseille, Institut Fresnel, Marseille, France
}

(Received 7 May 2021; revised 27 August 2021; accepted 30 September 2021; published 28 October 2021)

\begin{abstract}
We use balanced optical low-coherence interferometry to measure the ultralow light power backreflected and/or back-scattered by optical interfaces. Indeed, backscattered light from optical surfaces can be a critical source of noise in interferometric gravitational-wave detectors, such as the Laser Interferometer Gravitational-Wave Observatory (LIGO), Virgo, or the Laser Interferometer Space Antenna (LISA), and therefore, it is required to characterize, with high sensitivity, the scattering properties of elementary components involved in their optical design. The use of a broadband light source makes an easy identification of the scattering interface possible, while the implementation of balanced interferometric detection allows very low light levels to be detected, thanks to the simultaneous use of coherent gain and source intensity noise suppression. Experimental results are reported, first on bare glass optical windows (N-BK7 and S-LAH66) and then on a silver-coated mirror, that demonstrate the ability of this setup to quantify specular- or diffuse-reflectance coefficients as low as $10^{-11}$.
\end{abstract}

DOI: 10.1103/PhysRevApplied.16.044055

\section{INTRODUCTION}

Since the direct detection of gravitational waves (GWs) in 2015 by the Laser Interferometer Gravitational-Wave Observatory (LIGO) [1], several projects for the development of dedicated GW antennas have been launched, either for terrestrial implementation (Einstein Telescope, ET) or for deployment in space (Laser Interferometer Space Antenna, LISA). For all these interferometric instruments, scattered light is a critical issue [2-10] because, to a first approximation, it can induce phase-measurement errors that are directly influenced by the relative amplitude of these parasitic optical fields. By way of example, this means that phase uncertainties in the order of a few microradians can be induced by ultraweak retroreflected or back-scattered waves, the relative power of which is about $10^{-12}$. The availability of characterization equipment capable of detecting such ultralow light power is therefore a key point in any project for the development of GW antennas [11-13].

For such a characterization setup, the use of interferometric detection, either homodyne or heterodyne, is important because the only part of parasitic light that needs to be measured is that with a unitary overlapping integral with the single-mode illumination field. Moreover, the coherent gain provided by an interferometric detection scheme is a key point in attaining the required sensitivity, since, in such a scheme, measurement of the back-reflectance,

*michel.lequime@fresnel.fr $\rho$, is replaced by that of $\sqrt{\rho}$, the square root of this back-reflectance. However, this interferometric detection also has some drawbacks, similar to those occurring in gravitational-wave detectors, i.e., sensitivity to parasitic light, but it is also very difficult to identify the optical element responsible for any signal recorded by the interferometric receiver. To overcome these two difficulties, it is, in general, necessary to implement very efficient beam dumps and to apply a periodic displacement to the sample [14-16] to isolate its contribution within the coherent noise floor.

To overcome these two practical constraints, a very effective way is to implement low-coherence (or whitelight) interferometry, i.e., to replace the laser emitter used in the interferometric setup by a broadband source. One of the best-known examples of this approach is certainly the fiber-optic gyroscope, in which the use of such a broadband source almost cancels out the Rayleigh-scattering contribution generated throughout the length of the fiber [17]. The spatial selectivity provided by this "incoherent" scheme is also the starting point for the development of a method for characterizing optical waveguides: optical low-coherence reflectometry $[18,19]$. The same approach is used in optical coherence tomography to provide in vivo cross-section imaging capabilities with micrometer resolution, in particular, for retinal structures [20]. Different ways of implementing this general technique have been introduced and compared, focusing on either the ranging scheme (time domain, Fourier domain, swept source) [21] or the interferometric receiver structure (unbalanced, 
balanced) [22]. The simultaneous choice of time domain and unbalanced detection leads to very simple setups (Michelson interferometer); their sensitivity is mainly limited by the relative intensity noise of the source, and in practice it is difficult to exceed a dynamic range of $65 \mathrm{~dB}$ [23].

Here, we show how the replacement of an unbalanced interferometric detection by a balanced one allows this dynamic range to be improved to $110 \mathrm{~dB}$, by retaining all the advantages of low-coherence reflectometry. The paper is organized as follows: Sec. II provides a general description of the experimental setup, while Sec. III A provides a theoretical description of the signals recorded in the retroreflection regime and describes their numerical processing. Section III B presents the experimental measurement of ultralow light power corresponding to high-order multiple retroreflections in two different bare glass windows (N-BK7 and S-LAH66). Section IV follows the same organization (Sec. IV A experimental setup, Sec. IV B theoretical considerations, Sec. IV C experimental results) for back-scattering measurements made on a silver-coated mirror. Section V concludes this presentation and defines possible ways to improve the sensitivity of this equipment.

\section{EXPERIMENTAL SETUP}

Figure 1 shows the optical layout of our experimental setup. The light source is a fiber pigtailed superluminescent diode (SLD) from Thorlabs (1050S-A60), with a power of $60 \mathrm{~mW}$, a central wavelength about $1050 \mathrm{~nm}$, and a spectral width of $70 \mathrm{~nm}$. The pigtail consists of a Corning HI 1060 single-mode fiber (mode-field diameter $2 w_{0}=6.2 \mu \mathrm{m}$ ), the output of which is located in the focal plane of an offaxis parabolic mirror reflective collimator (RC) with an effective focal length, $f$, of $7 \mathrm{~mm}$ (Thorlabs RC02APC$\mathrm{P} 01)$. This optical assembly delivers a Gaussian collimated light beam with a waist diameter, $2 w_{f}$, of approximately $1.5 \mathrm{~mm}$ and a divergence, $\theta_{f}$, of approximately $0.45 \mathrm{mrad}$. After reflection from a silver mirror, $M_{1}\left(R_{M 1}=0.972\right)$,

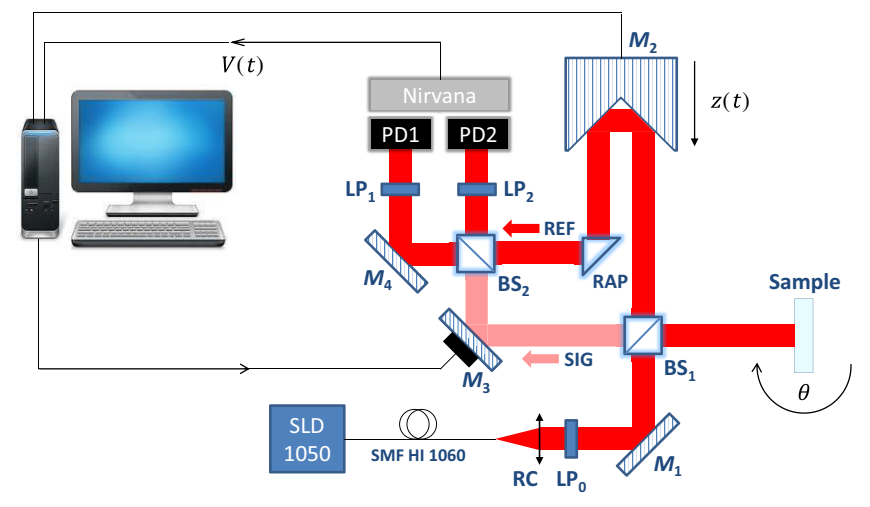

FIG. 1. Optical layout of the experimental setup. this main beam is split using a 50:50 cube beam splitter $\left(\mathrm{BS}_{1}\right)$ into the reference $\left(T_{1}=0.445\right)$ and signal $\left(R_{1}=\right.$ $0.459)$ arms of a Michelson-type interferometer. The signal arm consists of the sample mounted on a Thorlabs PRMTZ8 motorized rotation stage. The beam reflected by (or scattered from) the sample crosses the $\mathrm{BS}_{1}$ beam splitter $\left(T_{1}=0.445\right)$, and is directed towards a second beam splitter $\left(\mathrm{BS}_{2}, T_{2}=0.448, R_{2}=0.456\right)$ by a piezoactuated mirror, $M_{3}\left(R_{M 3}=0.972\right)$. The reference arm consists of a Thorlabs HRR201-P01 hollow retroreflector $\left(M_{2}, R_{M 2}=\right.$ 0.918 ) mounted on a Newport XMS100-S motorized linear translation stage. The use of a corner cube in the reference arm allows the reflected beam to be decoupled from the incident path and a right-angle prism (RAP, $R_{P}=$ 0.994 ) is used to direct this decoupled beam towards the 50:50 cube beam splitter, $\mathrm{BS}_{2}$, that recombines both signal and reference arms. The two beams provided by this last beam splitter are directed towards the two indium gallium arsenide (InGaAs) photodiodes (detector diameter $2 a=1$ $\mathrm{mm}$, responsivity $S=0.82 \mathrm{~A} / \mathrm{W}$ at $1064 \mathrm{~nm}$ ) of a Nirvana 2017 balanced optical receiver [24] from Newport. As a result of the propagation of the light beam over a distance about $115 \mathrm{~cm}$ from the reflective collimator RC, its modal diameter, $2 w_{d}$, at the detector is approximately 1.8 $\mathrm{mm}$, i.e., greater than the useful area of the photodiodes. The consequences of this point will be analyzed in Sec. III A [see Eq. (16)]. The piezoactuated mirror, $M_{3}$, is only used to fine-tune the alignment between reference and signal arms by maximizing the amplitude of the interference signal and remains static during measurements. The voltage, $V$, corresponding to the linear output channel of the Nirvana receiver and resulting from amplification of the difference between the photocurrents of the two photodiodes is digitized by a National Instruments (NI) acquisition module, USB-6212 (16 bits, $100 \mathrm{kS} / \mathrm{s}$ ). Moreover, a linear polarizer, $\mathrm{LP}_{0}$ (vertical polarization axis), is inserted just after the RC; this decreases the effective light power in the setup by a factor slightly greater than $2\left(T_{\mathrm{LP} 0}=0.413\right)$, but the implementation of two additional linear polarizers, $\mathrm{LP}_{1}$ and $\mathrm{LP}_{2}\left(T_{\mathrm{LP} 1}=T_{\mathrm{LP} 2}=0.826\right.$, vertical polarization axis in nominal configuration), inserted just before the two photodiodes of the Nirvana receiver, allows the balance between the two photodetectors to be finely tuned.

\section{LIGHT POWER BACK-REFLECTED BY BARE GLASS WINDOWS}

As highlighted in Sec. I, the final objective of this experimental system is to accurately measure the amount of light coherently back-scattered by optical components. However, to reach such a goal, it is important to first qualify the method on simpler cases for which a theoretical prediction of the expected result can be easily obtained. This procedure is very effective in determining the metrological 
performances of a new piece of equipment and to identify its possible limitations. This path is similar to that followed during the development of SALSA (for spectral and angular light-scattering characterization apparatus), a spectrophotometric instrument able to perform ultrawiderange measurements of the spectral transmittance of thinfilm filters (optical density up to 12) over the visible and infrared spectrum [25,26]; this is also used today to make wavelength- and angle-resolved scattering measurements of complex filters [27].

\section{A. Theoretical considerations}

Let us assume that the sample is a bare glass window at normal incidence. The amplitudes of the electric fields associated with the reference and signal arms on each photodiode, labels $j=1,2$, respectively, are defined in the harmonic regime by [28]

$$
\begin{aligned}
& \mathcal{E}_{\mathrm{ref}, j}(f)=t_{\mathrm{ref}, j} \mathcal{E}_{i}(f) e^{i k L_{\mathrm{ref}}}, \\
& \mathcal{E}_{\mathrm{sig}, j}(f)=t_{\mathrm{sig}, j} r(f) \mathcal{E}_{i}(f) e^{i k L_{\mathrm{sig}},}
\end{aligned}
$$

where $\mathcal{E}_{i}(f)$ is the amplitude of the incoming light beam at frequency $f, L_{\text {ref }}\left(L_{\text {sig }}\right)$ is the physical length of the reference (signal) arm, $k$ is the wave vector $(k=2 \pi f / c)$, $t_{\text {ref }, j}=\sqrt{T_{\text {ref }, j}} e^{i \Phi_{\text {ref }, j}}\left(t_{\text {sig }, j}=\sqrt{T_{\text {sig }, j}} e^{i \Phi_{\text {sig }, j}}\right)$ is the complex transmission coefficient of the reference (signal) arm, and $r(f)$ is the coefficient of reflection from this bare glass window, as illustrated by Fig. 2 and defined by

$$
r(f)=\sum_{m=1}^{\infty} r_{m} e^{2 i k(m-1) n(f) d},
$$

where $n(d)$ is the refractive index (thickness) of the window, and $r_{m}$ is given by [23]

$$
r_{m}=\begin{array}{ll}
\frac{1-n}{1+n} & m=1, \\
\frac{4 n(n-1)^{2 m-3}}{(n+1)^{2 m-1}} & \forall m \geqslant 2 .
\end{array}
$$

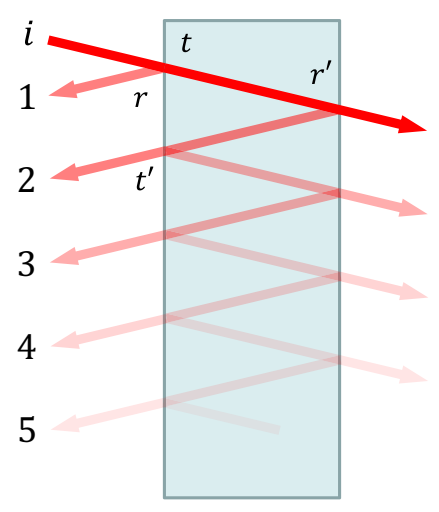

FIG. 2. Multiple reflections within a bare glass window.
The transmittance and phase quantities in Eq. (1) are given, for photodiode $j=1$, by

$$
\begin{aligned}
T_{\mathrm{ref}, 1}= & T_{\mathrm{LP} 0} R_{M 1} T_{1} R_{M 2} R_{P} T_{2} R_{M 4} T_{\mathrm{LP} 1}, \\
\Phi_{\mathrm{ref}, 1}= & k\left(n_{\mathrm{BS}}-1\right)\left(e_{1}+e_{2}\right)+k\left(n_{P}-1\right) e_{P}+\tau_{1}+\rho_{M 2} \\
& +\rho_{P}+\tau_{2}, \\
T_{\mathrm{sig}, 1}= & T_{\mathrm{LP} 0} R_{M 1} R_{1} T_{1} R_{M 3} R_{2} R_{M 4} T_{\mathrm{LP} 1}, \\
\Phi_{\mathrm{sig}, 1}= & k\left(n_{\mathrm{BS}}-1\right)\left(2 e_{1}+e_{2}\right)+\rho_{1}+\tau_{1}+\rho_{M 3}+\rho_{2},
\end{aligned}
$$

and, for photodiode $j=2$, by

$$
\begin{aligned}
T_{\text {ref }, 2}= & T_{\mathrm{LP} 0} R_{M 1} T_{1} R_{M 2} R_{P} R_{2} T_{\mathrm{LP} 2}, \\
\Phi_{\text {ref }, 2}= & k\left(n_{\mathrm{BS}}-1\right)\left(e_{1}+e_{2}\right)+k\left(n_{P}-1\right) e_{P}+\tau_{1} \\
& +\rho_{M 2}+\rho_{P}+\rho_{2}, \\
T_{\text {sig }, 2}= & T_{\mathrm{LP} 0} R_{M 1} R_{1} T_{1} R_{M 3} T_{2} T_{\mathrm{LP} 2}, \\
\Phi_{\text {sig }, 2}= & k\left(n_{\mathrm{BS}}-1\right)\left(2 e_{1}+e_{2}\right)+\rho_{1}+\tau_{1}+\rho_{M 3}+\tau_{2},
\end{aligned}
$$

$n_{\mathrm{BS}}\left(n_{P}\right)$ is the refractive index of the beam splitters (rightangle prism); $e_{j}\left(e_{p}\right)$ is the physical thickness of the beam splitter $j ; j=1,2$ (right-angle prism); and $\rho_{c}\left(\tau_{c}\right)$ is the phase change induced by the reflection from (transmission through) each component.

The electromagnetic field, $\mathcal{E}_{j}(f)$, at the center of each photodiode, $j$, is given by

$$
\mathcal{E}_{j}(f)=\mathcal{E}_{\mathrm{sig}, j}(f)+\mathcal{E}_{\mathrm{ref}, j}(f),
$$

which leads to the following expression for the electric currents provided by each photodetector:

$$
\begin{aligned}
I_{j} & =2 \pi \int_{0}^{a} e^{-2\left(u^{2} / w_{d}^{2}\right)} u d u \frac{c}{2 \pi} \int_{0}^{\infty} S(f)\left|\mathcal{E}_{j}(f)\right|^{2} d f, \\
& =\frac{\pi w_{d}^{2}}{2}\left[1-e^{2\left(a^{2} / w_{d}^{2}\right)}\right] \frac{c}{2 \pi} \int_{0}^{\infty} S(f)\left|\mathcal{E}_{j}(f)\right|^{2} d f,
\end{aligned}
$$

where $c$ is the speed of light.

If we assume that the light-power spectral density of the main beam is characterized by a Gaussian profile centered at $f=f_{0}$ (half-width $\delta f$ ), i.e.,

$$
\frac{\pi w_{d}^{2}}{2} \frac{c}{2 \pi}\left|\mathcal{E}_{i}(f)\right|^{2}=P_{i}(f)=\frac{P_{0}}{\delta f \sqrt{\pi}} e^{-\left[\left(f-f_{0}\right) / \delta f\right]^{2}},
$$

we obtain the following expression for the photodiode currents:

$$
\begin{aligned}
I_{j}= & \frac{P_{0}}{\delta f \sqrt{\pi}}\left[1-e^{2\left(a^{2} / w_{d}^{2}\right)}\right] \int_{0}^{\infty} S(f) e^{-\left[\left(f-f_{0}\right) / \delta f\right]^{2}} \\
& \times\left|t_{\mathrm{ref}, j} e^{i k L_{\mathrm{ref}}}+t_{\mathrm{sig}, j} e^{i k L_{\mathrm{sig}}} r(f)\right|^{2} d f .
\end{aligned}
$$


In the integral of Eq. (9), the modulus squared can be transformed as follows:

$$
\begin{aligned}
|\ldots|^{2}= & T_{\text {ref }, j}+|r(f)|^{2} T_{\text {sig }, j} \\
& +2 \sqrt{T_{\text {ref }, j} T_{\text {sig }, j}} \operatorname{Re}\left[r(f) e^{i\left(k \Delta L+\Delta \Phi_{j}\right)}\right],
\end{aligned}
$$

where $\Delta L$ and $\Delta \Phi_{j}$ are defined by

$$
\begin{aligned}
& \Delta L=L_{\mathrm{sig}}-L_{\mathrm{ref}}, \\
& \Delta \Phi_{j}=\Phi_{\mathrm{sig}, j}-\Phi_{\mathrm{ref}, j},
\end{aligned}
$$

i.e., using beam splitters and a right-angle prism with the same glass (fused silica, $\left.n_{\mathrm{BS}}=n_{P}\right)$ and size $\left(e_{1}=\right.$ $\left.e_{2}=e_{P}\right)$,

$$
\begin{aligned}
\Delta \Phi_{1} & =\rho_{1}+\rho_{2}-\tau_{2}+\rho_{M 3}-\rho_{M 2}-\rho_{P}, \\
\Delta \Phi_{2} & =\rho_{1}-\rho_{2}+\tau_{2}+\rho_{M 3}-\rho_{M 2}-\rho_{P}, \\
& =\Delta \Phi_{1}-2\left(\rho_{2}-\tau_{2}\right), \\
& =\Delta \Phi_{1}-\pi .
\end{aligned}
$$

The last identity is obtained using the general phaseclosure relation of a symmetric beam splitter [28]. We also note that the use of a right-angle prism in the reference arm (instead of a standard reflective mirror) allows the spectral dependence of the phase difference to be canceled. If, in addition, we neglect the spectral dependence of responsivity, $S$, and transmittance, $T_{\text {ref }, j}$ and $T_{\text {sig. }, j}$, over the source bandwidth, Eq. (9) becomes

$$
I_{j}=I_{\mathrm{dc}, j}+(-1)^{j+1} I_{\mathrm{ac}, j},
$$

where

$$
\begin{aligned}
I_{\mathrm{dc}, j}= & \eta_{a} T_{\mathrm{ref}, j} S\left(f_{0}\right) P_{0}+\eta_{a} T_{\mathrm{sig}, j} S\left(f_{0}\right) \frac{P_{0}}{\delta f \sqrt{\pi}} \\
& \times \int_{0}^{\infty} e^{-\left[\left(f-f_{0}\right) / \delta f\right]^{2}}|r(f)|^{2} d f, \\
I_{\mathrm{ac}, j}= & 2 \eta_{a} \sqrt{T_{\mathrm{ref}, j} T_{\mathrm{sig}, j}} S\left(f_{0}\right) \frac{P_{0}}{\delta f \sqrt{\pi}} \mathrm{Re} \\
& \times\left\{\int_{0}^{\infty} e^{-\left[\left(f-f_{0}\right) / \delta f\right]^{2}} r(f) e^{i\left(k \Delta L+\Delta \Phi_{1}\right)} d f\right\},
\end{aligned}
$$

and

$$
\eta_{a}=1-e^{2\left(a^{2} / w_{d}^{2}\right)}
$$

Assuming that the coherence length, $l_{c}=c / \pi \delta f$, of the SLD is much smaller than twice the optical thickness, $n d$, of the glass window, Eqs. (14) and (15) can be rewritten as
$[23,29]$

$$
\begin{aligned}
I_{\mathrm{dc}, j} & =\eta_{a} S\left(f_{0}\right) P_{0}\left(T_{\mathrm{ref}, j}+\frac{2 R}{1+R} T_{\mathrm{sig}, j}\right), \\
R & =\left(\frac{n-1}{n+1}\right)^{2},
\end{aligned}
$$

$$
\begin{aligned}
I_{\mathrm{ac}, j}= & 2 \eta_{a} \sqrt{T_{\mathrm{ref}, j} T_{\mathrm{sig}, j}} S\left(f_{0}\right) P_{0} \sum_{m=1}^{\infty} r_{m} \sqrt{K_{m}} e^{-\left(\pi K_{m} \Delta_{m} \delta f / c\right)^{2}} \\
& \times \cos \left(k_{0} \Delta_{m}+\Psi_{m}\right)
\end{aligned}
$$

where $k_{0}$ is the wave vector at the central frequency $f_{0}$, and $\Delta_{m}, K_{m}$, and $\Psi_{m}$ are defined by

$$
\begin{aligned}
\Delta_{m} & =\Delta L+2(m-1) d\left[n\left(f_{0}\right)+\left.f_{0} \frac{\partial n}{\partial f}\right|_{f_{0}}\right], \\
& =\Delta L+2(m-1) d n_{g}\left(f_{0}\right),
\end{aligned}
$$

$$
\begin{aligned}
K_{m} & =\frac{1}{\sqrt{1+D_{m}^{2}}}, \\
D_{m} & =2 \pi \frac{\delta f^{2}}{c}(m-1) d\left(\left.2 \frac{\partial n}{\partial f}\right|_{f_{0}}+\left.f_{0} \frac{\partial^{2} n}{\partial f^{2}}\right|_{f_{0}}\right), \\
\Psi_{m} & =\frac{1}{2} \arctan \left(D_{m}\right)+D_{m}\left(\pi K_{m} \Delta_{m} \delta f / c\right)^{2} .
\end{aligned}
$$

The voltage, $V$, provided by the Nirvana receiver on its linear output channel corresponds to the amplification of the difference between the two photocurrents, $I_{1}$ and $I_{2}$, after fine-tuning the balance between their dc components. This balance is achieved via a small rotation $\left(\beta \approx 10^{\circ}\right)$ of the linear polarizer $\mathrm{LP}_{2}\left(T_{\mathrm{LP} 2} \rightarrow T_{\mathrm{LP} 2} \cos ^{2} \beta\right)$. Consequently, using Eq. (13),

$$
V=G\left(I_{1}-\cos ^{2} \beta I_{2}\right)=G\left(I_{\mathrm{ac}, 1}+\cos ^{2} \beta I_{\mathrm{ac}, 2}\right),
$$

i.e., using Eq. (18),

$$
\begin{aligned}
& V=2 \eta_{a}\left(\sqrt{T_{\mathrm{ref}, 1} T_{\mathrm{sig}, 1}}+\cos ^{2} \beta \sqrt{T_{\mathrm{ref}, 2} T_{\mathrm{sig}, 2}}\right) \\
& G S\left(f_{0}\right) P_{0} \sum_{m=1}^{\infty} r_{m} \sqrt{K_{m}} e^{-\left(\pi K_{m} \Delta_{m} \delta f / c\right)^{2}} \cos \left(k_{0} \Delta_{m}+\Psi_{m}\right),
\end{aligned}
$$

where $G$ is the transimpedance gain of the Nirvana receiver in balanced mode $\left(10^{5} \mathrm{~V} / \mathrm{A}\right)$.

As indicated before, the hollow retroreflector used in the reference arm of the interferometer is mounted on 
an XMS100-S translation stage. This equipment is driven by an ironless linear motor, which enables ultraprecise translation motion with exceptional speed stability (better than $0.05 \%$ ) and speeds of up to $300 \mathrm{~mm} / \mathrm{s}$. The difference in length between the two arms of the interferometer, therefore, becomes a linear function of time, i.e.,

$$
L_{\mathrm{ref}}-L_{\mathrm{sig}}=2\left(z-z_{1}\right)=2 v\left(t-t_{1}\right),
$$

where $v$ is the translation speed of the stage and $t_{1}$ is the time for which the lengths of the two arms are equal. This means that all optical path differences (OPDs), $\Delta_{m}$, can be written as

$$
\Delta_{m}=2 v\left(t-t_{m}\right), \quad \text { where } t_{m}=t_{1}+\frac{(m-1) n_{g} d}{v},
$$

and that the time dependence of the voltage is characterized by the presence of a carrier frequency, $f_{c}$, defined by

$$
f_{c}=\frac{2 v}{\lambda_{0}}
$$

Figure 3 shows this time dependence around $t_{1}$ (a) and over a longer duration (b), corresponding to the time, $t_{5}$, needed to record the first five echoes.

The sample is assumed to be a 1-mm-thick N-BK7 window. The displacement speed of the stage here is equal to $0.5 \mathrm{~mm} / \mathrm{s}$, while the coherence length of the source corresponds to that of the SLD used in the experimental setup $(\Delta \lambda=70 \mathrm{~nm})$, i.e.,

$$
l_{c}=\frac{c}{\pi \delta f}=\frac{2 \sqrt{\ln 2}}{\pi} \frac{\lambda_{0}^{2}}{\Delta \lambda}=8.35 \mu \mathrm{m} .
$$

Only the first three echoes are clearly visible in Fig. 3(b); this is due to the very rapid decrease in the amplitude, $A_{m}$,
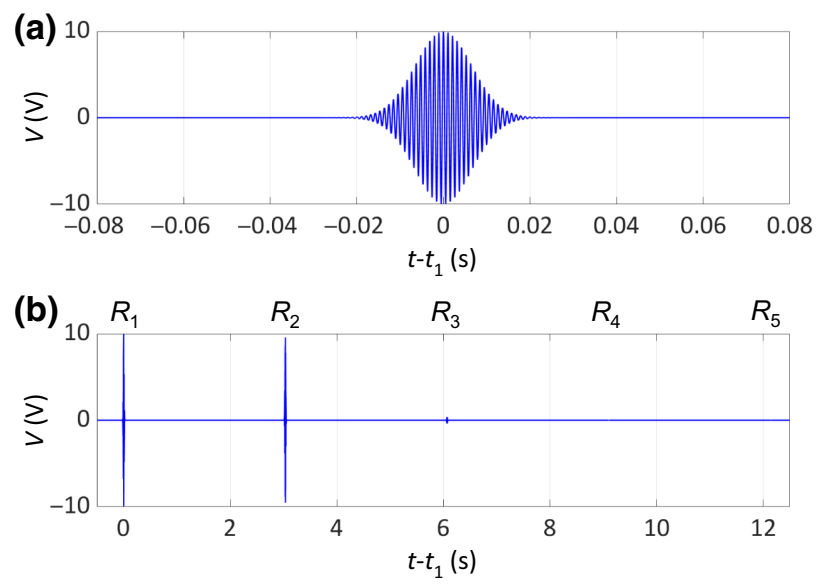

FIG. 3. Theoretical time dependence of the voltage provided by the output channel of the Nirvana receiver.
TABLE I. Multiple reflection coefficients and related echo amplitudes for the N-BK7 window.

\begin{tabular}{lccccc}
\hline \hline$m$ & 1 & 2 & 3 & 4 & 5 \\
\hline$R_{m}$ & 0.041 & 0.038 & $6.3 \times 10^{-5}$ & $1.0 \times 10^{-7}$ & $1.7 \times 10^{-10}$ \\
$\left|r_{m}\right|$ & 0.202 & 0.194 & $7.9 \times 10^{-3}$ & $3.2 \times 10^{-4}$ & $1.3 \times 10^{-5}$ \\
\hline \hline
\end{tabular}

of these echoes with their order number, $m\left(A_{m} \sim\left|r_{m}\right|=\right.$ $\sqrt{R_{m}}$ ), as summarized in Table I in the case of a N-BK7 window.

Therefore, it is important to identify a method for improving the signal-to-noise ratio of the voltage recordings and, then, to make the detection of not only high-order echoes $(m \geqslant 4)$, but also scattered light, possible. This is achieved through narrow-frequency filtering around the carrier frequency generated by the translation of the hollow retroreflector at constant speed. Indeed, as expected, the power spectrum of the time-dependent voltage exhibits two sharp peaks that correspond to the Fourier transform of the cosine term (see Fig. 4), and the broadening of these peaks induced by the small coherence length of the source is moderate enough to implement a tight filtering characterized by a full bandwidth of approximately $100 \mathrm{~Hz}$. The 3 -dB bandwidth of the Nirvana receiver is $125 \mathrm{kHz}$, so this frequency filtering provides an improvement in the signalto-noise ratio as the square root of the bandwidth ratio, i.e., 35 .

\section{B. Experimental results}

We record the light back-reflected by two different uncoated optical windows, i.e., 1-mm-thick N-BK7 [30] and 2-mm-thick S-LAH66 [31], using the experimental
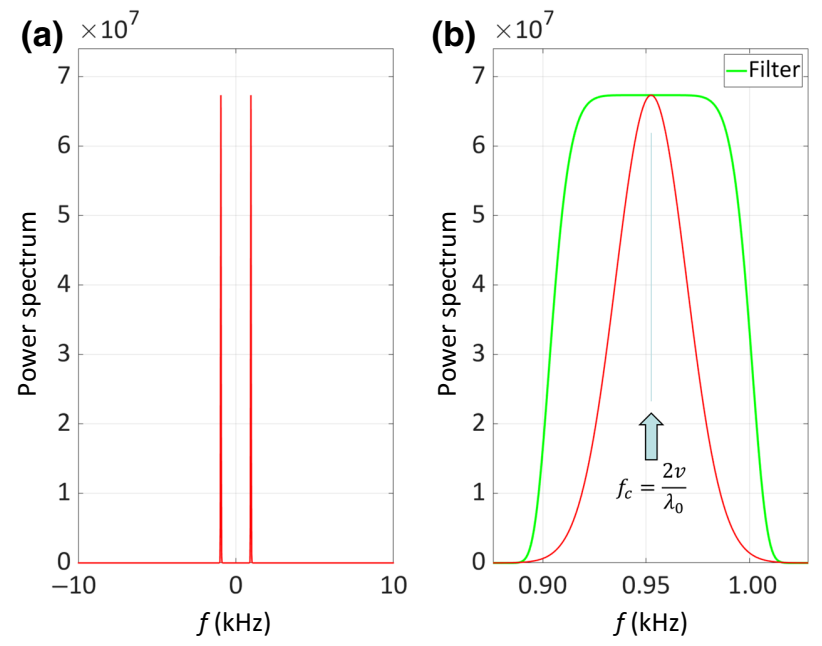

FIG. 4. Frequency filtering. (a) Power spectrum of voltage $V(t)$. (b) Enlarged view of this power spectrum around the carrier frequency. $f_{c}$ (red curve), and profile of the filter (green curve). 
TABLE II. Influence of the driving current on the characteristics of the superluminescent diode.

\begin{tabular}{lccc}
\hline \hline$I(\mathrm{~mA})$ & $P_{0}(\mathrm{~mW})$ & $\lambda_{0}(\mathrm{~nm})$ & $\Delta \lambda(\mathrm{nm})$ \\
\hline 160 & 2.0 & 1074.2 & 28.1 \\
180 & 4.9 & 1070.9 & 31.2 \\
1000 & 57.8 & 1044.0 & 83.6 \\
\hline \hline
\end{tabular}

setup described in Sec. II and data processing explained at the end of Sec. III A.

Before going on to present and discuss the experimental results we obtain, it is important to indicate that the light power detected by each photodiode of the Nirvana receiver must remain below $0.5 \mathrm{~mW}$, which is the photodetector saturation limit (saturation constraint). The presence of a dc term in Eq. (13) may require an adjustment to the source light power, at least in the reference arm, to satisfy this requirement. Moreover, we have to limit the maximum value of the Nirvana's output voltage to $\pm 10 \mathrm{~V}$, which corresponds to the digitization range of the NI acquisition module (digitizing-range constraint). A possible solution to these two problems would be to insert an optical density in the main arm, but, because of the large OPD scan during a back-reflection measurement, we prefer to avoid the possible generation of spurious echoes induced by additional optical interfaces in the setup. The conclusion would not be the same for a back-scattering measurement (see Sec. IV).

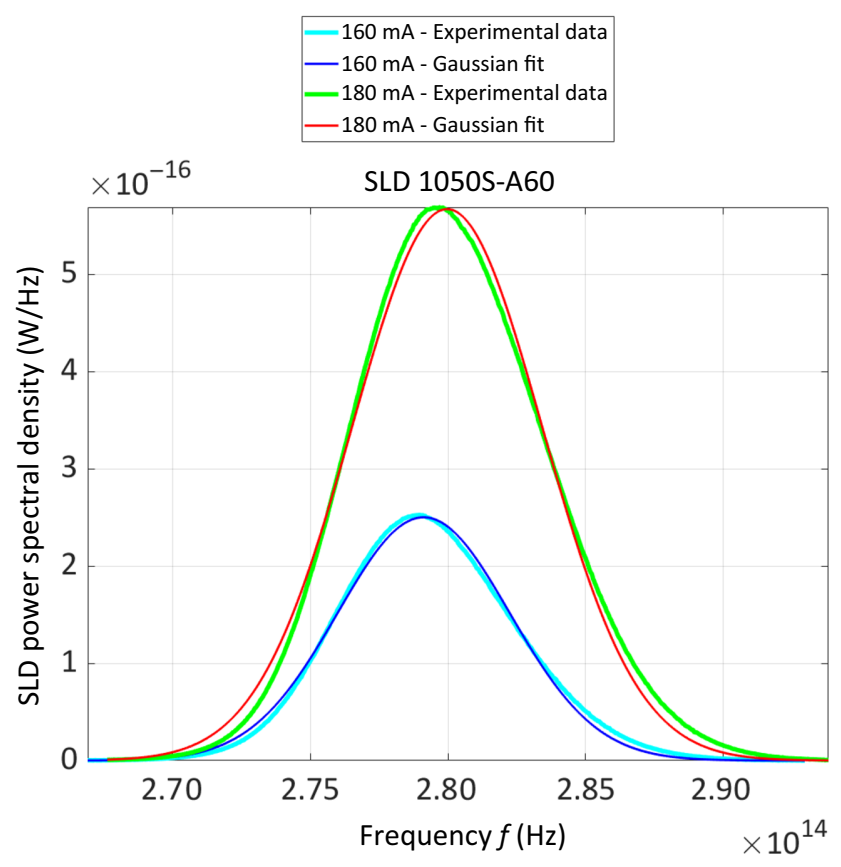

FIG. 5. SLD power spectral density for both driving currents (cyan curve, experimental data at $160 \mathrm{~mA}$; blue curve, Gaussian fit at $160 \mathrm{~mA}$; green curve, experimental data at $180 \mathrm{~mA}$; red curve, Gaussian fit at $180 \mathrm{~mA}$ ).
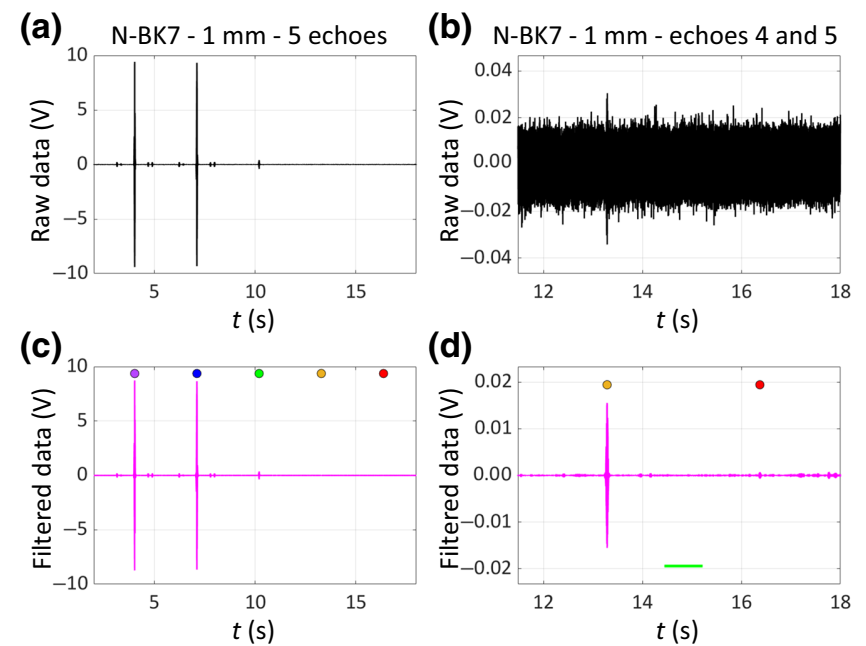

(d)

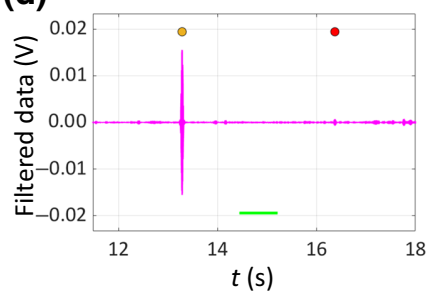

FIG. 6. Experimental measurement of the time dependence of voltage, $V$, recorded during translation of the hollow retroreflector at constant speed, $v$, of $0.5 \mathrm{~mm} / \mathrm{s}$ for the $1-\mathrm{mm}$-thick N-BK7 window.

Accordingly, for the measurement of back-reflected light, the source power is adjusted by decreasing the driving current of the superluminescent diode $(180 \mathrm{~mA}$ for the N-BK7 window, $160 \mathrm{~mA}$ for the S-LAH66 window, to be compared to a nominal full-power value of $1000 \mathrm{~mA}$ ). This power adjustment has a consequence on the central wavelength, $\lambda_{0}$, and spectral bandwidth, $\Delta \lambda$, of the source, as summarized in Table II. The narrowing of the SLD spectral bandwidth allows frequency filtering in the Fourier space to be improved (full bandwidth reduced to approximately $50 \mathrm{~Hz}$ ). Moreover, Fig. 5 shows, for both driving currents, a comparison between the experimentally recorded power spectral density (PSD) and a Gaussian fit. The agreement is very satisfactory.
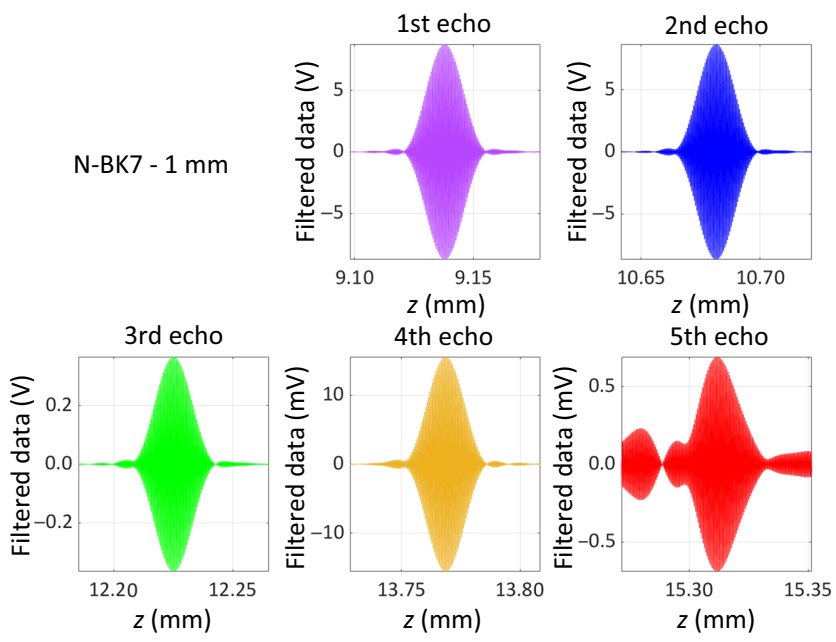

FIG. 7. Enlarged views of the five detected echoes for the 1-mm-thick N-BK7 window. 

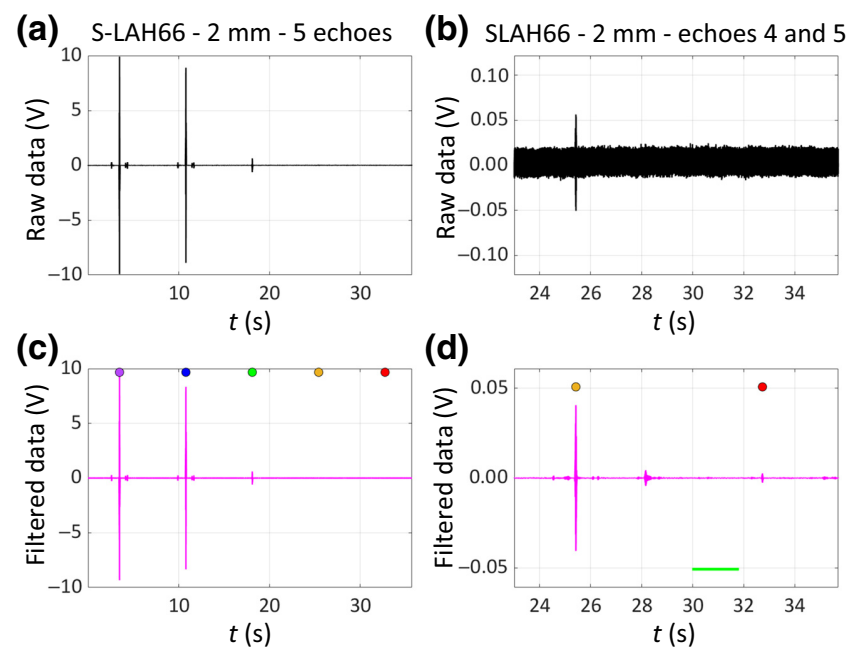

(d)

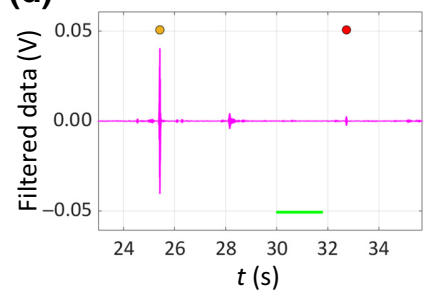

FIG. 8. Time dependence of voltage, $V$, for the 2-mm-thick SLAH66 window.

The measurement results corresponding to the detection of the five echoes are shown in Figs. 6 and 7 for the N-BK7 window and in Figs. 8 and 9 for the S-LAH66 window.

For each type of window, the first figure shows the time dependence of the voltage, $V$, recorded during translation of the hollow retroreflector at a constant speed, $v$, of $0.5 \mathrm{~mm} / \mathrm{s}$ (OPD scan), while the second figure presents enlarged views of the five detected echoes. In the first figures, graph (a) [(c)] corresponds to raw (filtered) data recorded during the whole scan, while graph (b) [(d)] corresponds to an enlarged view of those recorded during the last part of the OPD scan (approximately from $2 \mathrm{~s}$ before $t_{4}$ up to $2 \mathrm{~s}$ after $t_{5}$ ). One can clearly see in these last two graphs the great improvement in the signal-to-noise ratio induced by frequency filtering.
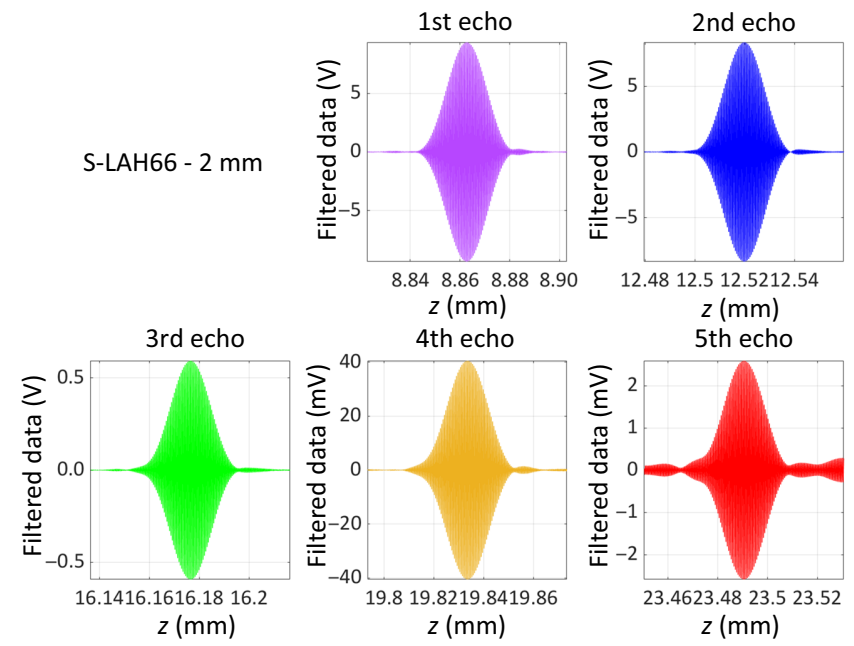

FIG. 9. Enlarged views of the five detected echoes for the 2mm-thick S-LAH66 window.
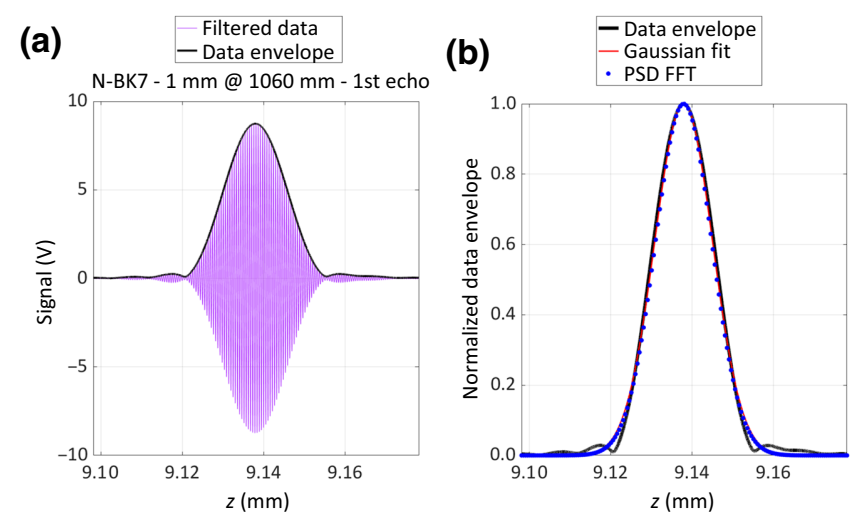

FIG. 10. Signal envelope extraction and modeling. (a) First echo recorded on a 1-mm-thick N-BK7 window (violet curve); data envelope obtained using a Hilbert transform (black curve). (b) Comparison between the normalized data envelope (black curve), a Gaussian fit (red curve), and the result of a Fourier transform of the SLD PSD Gaussian fit (blue circles).

To estimate the metrological quality of these measurements, we have to make a precise comparison between the experimentally recorded amplitude and position of these five echoes and those resulting from the theoretical approach presented in Sec. III A. To this end, we start by calculating the signal envelope of recorded voltage, $V$, using a Hilbert transform [32,33]. Figure 10(a) shows an example of the results obtained for the first echo recorded on the N-BK7 window (black curve).

To confirm the validity of the modeling of spectral and spatial signals using Gaussian distributions (already highlighted in Fig. 5), Fig. 10(b) shows a comparison between the calculated data envelope (black curve), a Gaussian fit (red curve) that is defined by

$$
E_{1}(z)=A_{1} e^{-\left[\left(z-z_{1}\right) / W_{1}\right]^{2}}
$$

and the Fourier transform of the Gaussian fit of the PSD source (blue circles). Again, the agreement is very satisfactory. By applying such an approach (envelope extraction followed by Gaussian fitting) to all echoes recorded on a glass window, we can determine the absolute amplitude, $A_{m}$, of each echo; its spatial width, $W_{m}$; and the position, $z_{m}$, of the translation stage that corresponds to the center of the Gaussian fit.

TABLE III. Accuracy of the measurement of distance $\delta z_{m}$ between two consecutive echoes. The group index, $n_{g}\left(f_{0}\right)$, calculated from manufacturer's data is used to estimate the physical thickness of the window.

\begin{tabular}{lccc}
\hline \hline Glass & $\left\langle\delta z_{m}\right\rangle(\mu \mathrm{m})$ & $n_{g}\left(f_{0}\right)$ & $d(\mathrm{~mm})$ \\
\hline N-BK7 & $1543.6 \pm 0.2$ & 1.5168 & 1.018 \\
S-LAH66 & $3657.0 \pm 0.2$ & 1.7763 & 2.059 \\
\hline \hline
\end{tabular}


TABLE IV. Comparison between experimental and theoretical values of $K_{m}$ and $R_{m}$ for the first five echoes recorded on the N-BK7 window.

\begin{tabular}{lcccccc}
\hline \hline$m$ & $A_{m}(\mathrm{~V})$ & $W_{m}(\mu \mathrm{m})$ & $K_{m}^{\text {expt. }}$ & $K_{m}^{\text {theor }}$ & $R_{m}^{\text {expt. }}$ \\
\hline 1 & 8.7401 & 10.072 & 1.0000 & 1.0000 & $3.4 \times 10^{-2}$ & $R_{m}^{\text {theor }}$ \\
2 & 8.6640 & 10.075 & 0.9997 & 1.0000 & $3.3 \times 10^{-2}$ & $3.7 \times 10^{-2}$ \\
3 & 0.3670 & 10.142 & 0.9932 & 0.9999 & $6.0 \times 10^{-5}$ & $6.2 \times 10^{-5}$ \\
4 & 0.0156 & 10.122 & 0.9951 & 0.9998 & $1.1 \times 10^{-7}$ & $1.0 \times 10^{-7}$ \\
5 & 0.0007 & 11.817 & 0.8523 & 0.9997 & $2.3 \times 10^{-10}$ & $1.7 \times 10^{-10}$ \\
\hline \hline
\end{tabular}

Let us consider first the positions, $z_{m}$, of the five echoes. In accordance with Eqs. (19) and (24), we have

$$
\delta z_{m}=\frac{1}{2}\left(\Delta_{m+1}-\Delta_{m}\right)=n_{g}\left(f_{0}\right) d \quad \text { for } m=1 \text { to } 4 .
$$

Consequently, for each window, we have access to four independent determinations of the quantity $n_{g}\left(f_{0}\right) d$, from which we may derive an estimate of the accuracy of the measurement of the relative position of the first five echoes; this is summarized in Table III.

Consider now the amplitude, $A_{m}$, of the same five echoes. By combining (23) and (28), we may write

$$
\frac{A_{m}}{A_{1}}=\frac{\left|r_{m}\right| \sqrt{K_{m}}}{\left|r_{1}\right| \sqrt{K_{1}}}, \quad \frac{W_{m}}{W_{1}}=\frac{K_{1}}{K_{m}} .
$$

Moreover, the definition of $K_{m}$ [see Eq. (20)] means that $K_{1}=1$. Consequently, we have

$$
\frac{R_{m}}{R_{1}}=\frac{1}{K_{m}}\left[\frac{A_{m}}{A_{1}}\right]^{2},
$$

where $K_{m}$ can be estimated in two different ways: the first, theoretical, is based on Eq. (20), and the second, experimental, from the determination of the width, $W_{m}$, of the Gaussian envelope fit, namely,

$$
K_{m}=\frac{W_{1}}{W_{m}} .
$$

Finally, the variation of the ratio $R_{m} / R_{1}$ with the echo order, $m$, is theoretically given by [see Eq. (3)]

$$
\frac{R_{m}}{R_{1}}=\left(1-R_{1}\right)^{2} R_{1}^{2 m-4}
$$

which depends only on $R_{1}$. Accordingly, an independent estimate of $R_{1}$ can be obtained by fitting the logarithmic variation of this ratio with the echo order; from this, the value of all reflection coefficients may be derived. Tables IV and V summarize the results obtained for 1-mmthick N-BK7 and 2-mm-thick S-LAH66, respectively.

Note that the dispersion effects, directly connected with the increase in width, $W_{m}$, with the echo order, $m$, are too small in the case of N-BK7 to be accurately detected. Conversely, the agreement between experimental results and theoretical predictions on the related $K_{m}$ parameter is very satisfactory in the case of S-LAH66. This is due to a big increase in the amplitude of these dispersion effects: indeed, the second-order dispersion coefficient is 7 times larger, while the window is 2 times thicker.

With regard to the measurement of multiple reflection coefficients, the overall accuracy attained on both windows is approximately the same, in the range of $20 \%$, as illustrated by Fig. 11(color codes are the same as those used in Figs. 7 and 9). This accuracy is in accordance with that demonstrated by state-of-the-art scatterometers [34 36]. Indeed, for such ultralow values, it is not possible to compare the reported performance with that of standard spectrophotometers working in reflection mode. Finally, the noise-floor value given in Fig. $11\left(6 \times 10^{-11}\right.$, dashed line) corresponds to the lowest value of the coefficient of reflection that can be measured with such SLD driving currents (less than $180 \mathrm{~mA}$ ). It is determined as follows: the standard deviation, $\sigma_{V}$, of the linear output voltage is calculated on data recorded far from any echo [indicated by a green bar in graph (d) of Figs. 6 and 8]. The noise floor is

\begin{tabular}{|c|c|c|c|c|c|c|}
\hline$m$ & $A_{m}(\mathrm{~V})$ & $W_{m}(\mu \mathrm{m})$ & $K_{m}^{\text {expt. }}$ & $K_{m}^{\text {theor }}$ & $R_{m}^{\text {expt. }}$ & $R_{m}^{\text {theor }}$ \\
\hline 1 & 9.3447 & 10.560 & 1.0000 & 1.0000 & $9.5 \times 10^{-2}$ & $7.5 \times 10^{-2}$ \\
\hline 2 & 8.3449 & 10.635 & 0.9930 & 0.9963 & $7.6 \times 10^{-2}$ & $6.4 \times 10^{-2}$ \\
\hline 3 & 0.5937 & 10.737 & 0.9835 & 0.9853 & $3.9 \times 10^{-5}$ & $3.6 \times 10^{-4}$ \\
\hline 4 & 0.0406 & 11.011 & 0.9590 & 0.9678 & $1.9 \times 10^{-6}$ & $2.0 \times 10^{-6}$ \\
\hline 5 & 0.0026 & 11.006 & 0.9595 & 0.9448 & $7.7 \times 10^{-9}$ & $1.1 \times 10^{-8}$ \\
\hline
\end{tabular}
simply the value of the coefficient of reflection that would correspond to 3 times this standard deviation, $\sigma_{V}$.

TABLE V. Comparison between experimental and theoretical values of $K_{m}$ and $R_{m}$ for the first five echoes recorded on the S-LAH66 window. 


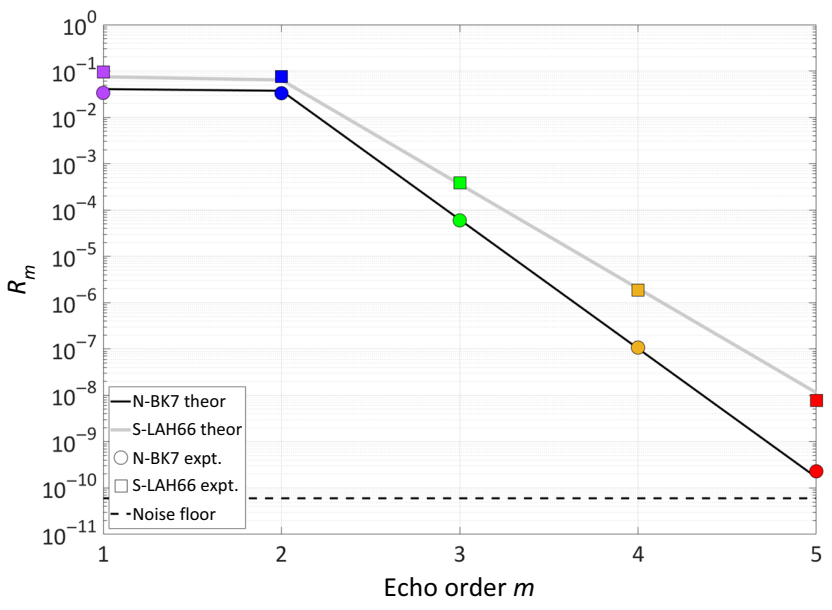

FIG. 11. Synthetic comparison between experimental results and theoretical data for both glass windows (for N-BK7: theoretical data, black line; experimental data, colored disks; for S-LAH66, theoretical data, gray line; experimental data, colored squares; noise floor, dashed line).

\section{LIGHT POWER BACK-SCATTERED FROM A SILVER-COATED MIRROR}

\section{A. Experimental setup}

The experimental results obtained on bare glass windows demonstrate that the setup we develop allows backreflected coefficients as low as $6 \times 10^{-11}(-102 \mathrm{~dB})$ to be measured.

To extend this method to record the angular distribution of light scattered by optical components, with a level between -95 and $-125 \mathrm{~dB}$, we need to implement the broadband source at its nominal driving current $(1000 \mathrm{~mA})$ and to eliminate any unnecessary loss of power in the setup. It is for this reason that we prefer to replace the optical density in the reference arm by a tunable splitting ratio between signal and reference beams, as shown in Fig. 12.

This is achieved by replacing the first nonpolarizing beam splitter, $\mathrm{BS}_{1}$, used in the initial setup (see Fig. 1) by a polarizing one (PBS, $T_{\mathrm{PBS}}=0.967$ and $R_{\mathrm{PBS}}=0.985$ ) and by adding an achromatic quarter-wave plate (QWP, $\left.T_{\mathrm{QWP}}=0.990\right)$ in the signal arm. If the passing direction of the linear polarizer, $\mathrm{LP}_{0}$, is oriented at $45^{\circ}$ to the vertical, $50 \%$ of the main beam is directed towards the sample and $50 \%$ towards the hollow retroreflector, as previously. Moreover, this splitting ratio can be continuously adjusted between $0 \%$ and $100 \%$ by simply rotating the linear polarizer, $\mathrm{LP}_{0}$.

The light beam reflected by the polarizing beam splitter is $S$-polarized. The slow and fast axes of the quarter-wave plate are oriented at $45^{\circ}$ of the vertical, and the beam transmitted by this birefringent plate is thus circularly polarized, e.g., right handed. The light beam reflected by the sample is again circularly polarized, but this time is left handed, and

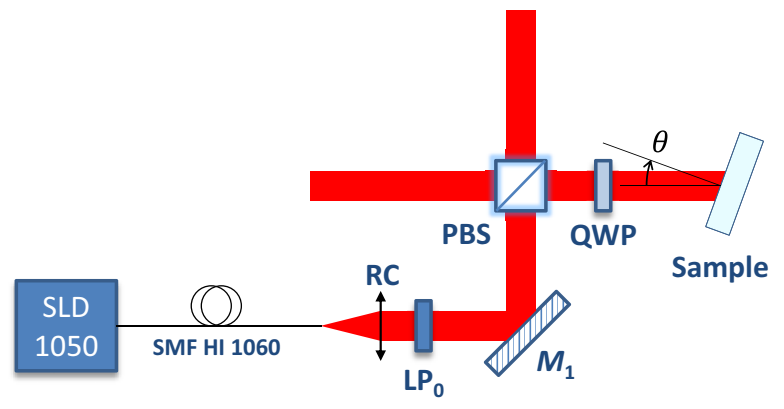

FIG. 12. Tunable-splitting-ratio scheme.

the reverse crossing of the quarter-wave plate transforms this polarization into a linear one, $P$-polarized, which is very efficiently transmitted by the polarizing beam splitter. Accordingly, Eq. (1) here becomes

$$
\begin{aligned}
& \mathcal{E}_{\mathrm{ref}, j}(f)=\sin \alpha t_{\mathrm{ref}, j}^{\mathrm{TSR}} \mathcal{E}_{i}(f) e^{i k L_{\mathrm{ref}}}, \\
& \mathcal{E}_{\mathrm{sig}, j}^{\mathrm{RR}}(f)=\cos \alpha t_{\mathrm{sig}, j}^{\mathrm{TSR}} r_{S}(f) \mathcal{E}_{i}(f) e^{i k L_{\mathrm{sig}},}
\end{aligned}
$$

where $\alpha$ is the angle between the polarization axis of $\mathrm{LP}_{0}$ and the vertical axis, and $r_{S}$ is the reflection coefficient of the front face of the sample. Superscript RR is used to indicate that we consider here the retroreflection on the front face of the sample, while superscript TSR underlines that we are implementing the tunable-splitting-ratio scheme described by Fig. 12. In this modified arrangement, the transmission coefficient of the reference arm, i.e., $t_{\text {ref }, j}^{\mathrm{TSR}}$, is identical to $t_{\text {ref }, j}$ [see Eqs. (4) and (5)], except that $T_{1}$ is replaced by $T_{\mathrm{PBS}}$. In the case of $t_{\mathrm{sig}, j}^{\mathrm{TSR}}$, we similarly have to replace $T_{1}\left(R_{1}\right)$ with $T_{\mathrm{PBS}}\left(R_{\mathrm{PBS}}\right)$, but also take into account the back-and-forth crossing of the quarter-wave plate (multiplication by $T_{\mathrm{QWP}}^{2}$ ).

\section{B. Theoretical considerations}

Actually, the presentation we make for a retroreflected beam, RR, is still valid for a back-scattered beam, BB: indeed, this back-scattered beam is located in the plane of incidence, and for low-roughness samples, this implies that an $S$-polarized beam produces $S$-polarized scattered light, and the same for $P$ polarization [28]. Consequently, we have

$$
\begin{aligned}
\mathcal{E}_{\mathrm{sig}, j}^{\mathrm{BS}}(f)= & \frac{\cos \alpha}{\sqrt{2}} t_{\mathrm{sig}, j}^{\mathrm{TSR}} \mathcal{E}_{i}(f) e^{4 i \pi n_{o} e f / c} e^{i k L_{\mathrm{sig}}} \\
& \times \sum_{j=0}^{p}\left[\left(C_{j, S S}^{-}+C_{j, P P}^{-}\right) \widehat{h}_{e, j}\left(-\vec{v}_{i}\right)\right],
\end{aligned}
$$

where $p$ is the number of layers involved in the coating of the substrate's front face; $C_{j, S S}^{-}$and $C_{j, P P}^{-}$are scattering coefficients that depend only on the angle of incidence and the structure of the stack (refractive indices and thicknesses 
of the layers); $\vec{v}_{i}$ is the tangential component of the incident wave vector $\left[v_{i}=(\sin \theta) / \lambda\right.$, where $\theta$ is the tilt angle of the sample about the vertical with respect to the direction of the illuminating beam (see Fig. 12)], and $\widehat{h}_{e, j}\left(v_{x}, v_{y}\right)$ is the Fourier transform of the effective two-dimensional surface profile of interface $j$, defined as the product of $h_{j}(x, y)$, the surface profile of this interface, and $s_{e}(x, y)$, the field distribution corresponding to this illuminating beam, i.e.,

$$
h_{e, j}(x, y)=h_{j}(x, y) e^{i k x \sin \theta} e^{-\left\{\left[(x \cos \theta)^{2}+y^{2}\right] / w_{s}^{2}\right\}},
$$

where $2 w_{s}$ is the modal diameter of the illuminating Gaussian beam on the sample $\left(2 w_{s}=1.57 \mathrm{~mm}\right)$.

The Fourier transform of $s_{e}$ is a Gaussian centered on $\vec{v}_{i}$, the spatial frequency width, $\delta v$, of which is very small, as given by

$$
\delta v=\frac{1}{\pi w_{s}} \approx 4.1 \times 10^{-4} \mu \mathrm{m}^{-1} .
$$

Therefore, using the properties of the Fourier transform, we have

$$
\widehat{h}_{e, j}(\vec{v})=\left[\widehat{h}_{j} \otimes \widehat{s}_{e}\right](\vec{v}) \simeq \widehat{h}_{j}(\vec{v}) \otimes \delta\left(\vec{v}-\vec{v}_{i}\right)=\widehat{h}_{j}\left(\vec{v}-\vec{v}_{i}\right)
$$

This allows the angle resolved back-scattering (ARBS) to be calculated, i.e.,

$$
\mathcal{B}_{S}(\theta)=\frac{\cos \theta}{4 S \lambda^{2}}\left|\sum_{j=0}^{p}\left[\left(C_{j, S S}^{-}+C_{j, P P}^{+}\right) \widehat{h}_{j}\left(-2 \vec{v}_{i}\right)\right]\right|^{2},
$$

where $S$ is the effective area of the sample, defined by the illumination condition, and given by

$$
S=\frac{\pi w_{s}^{2}}{2 \cos \theta}
$$

Suppose that the sample coating consists of only two layers [37], namely, a thick (200 nm) silver layer deposited by sputtering on the surface of a silica substrate and overcoated by a protective layer of silica $(113 \mathrm{~nm})$. We also assume that the roughness replication mechanism between interfaces is perfect, which allows Eq. (38) to be transformed into

$$
\mathcal{B}_{S}(\theta)=\frac{\cos \theta}{4 \lambda^{2}}\left|\sum_{j=0}^{2}\left(C_{j, S S}^{-}+C_{j, P P}^{+}\right)\right|^{2} \gamma\left(-2 v_{i}\right),
$$

where $\gamma$ is the roughness spectrum of the substrate

$$
\gamma(\vec{v})=\left.\frac{1}{S} \widehat{h}(\vec{v})\right|^{2} .
$$

Figure 13 (red curve) shows the result of a numerical computation of the angular dependence of light back-scattered by a protected silver mirror when illuminated by a circularly polarized beam, as described in Fig. 12. The roughness spectrum of the sample is replaced here by its polar mean, $\bar{\gamma}(v)$, which is assumed to be described by a standard Gaussian/exponential law [28,38], i.e.,

$$
\begin{aligned}
\bar{\gamma}(\nu)= & \pi\left(\delta_{g} L_{g}\right)^{2} e^{-\left(\pi L_{g} v\right)^{2}} \\
& +2 \pi\left(\delta_{e} L_{e}\right)^{2}\left[1+\left(2 \pi L_{e} v\right)^{2}\right]^{-3 / 2},
\end{aligned}
$$

where $\delta_{g}=4 \mathrm{~nm}, L_{g}=0.1 \mu \mathrm{m}, \delta_{e}=4 \mathrm{~nm}$, and $L_{e}=$ $2 \mu \mathrm{m}$.

The blue and green curves correspond to a thought experiment where the QWP is not present and the polarization state of the incoming light beam is linear and either aligned with or perpendicular to the passing direction of the polarizing beam splitter. Neglecting spectral dispersion effects, in particular, for the Fourier transform of the surface topography, and using the same approach as that detailed in Sec. III A, we can summarize the angular dependence of the intensity of the voltage, $V$, provided by

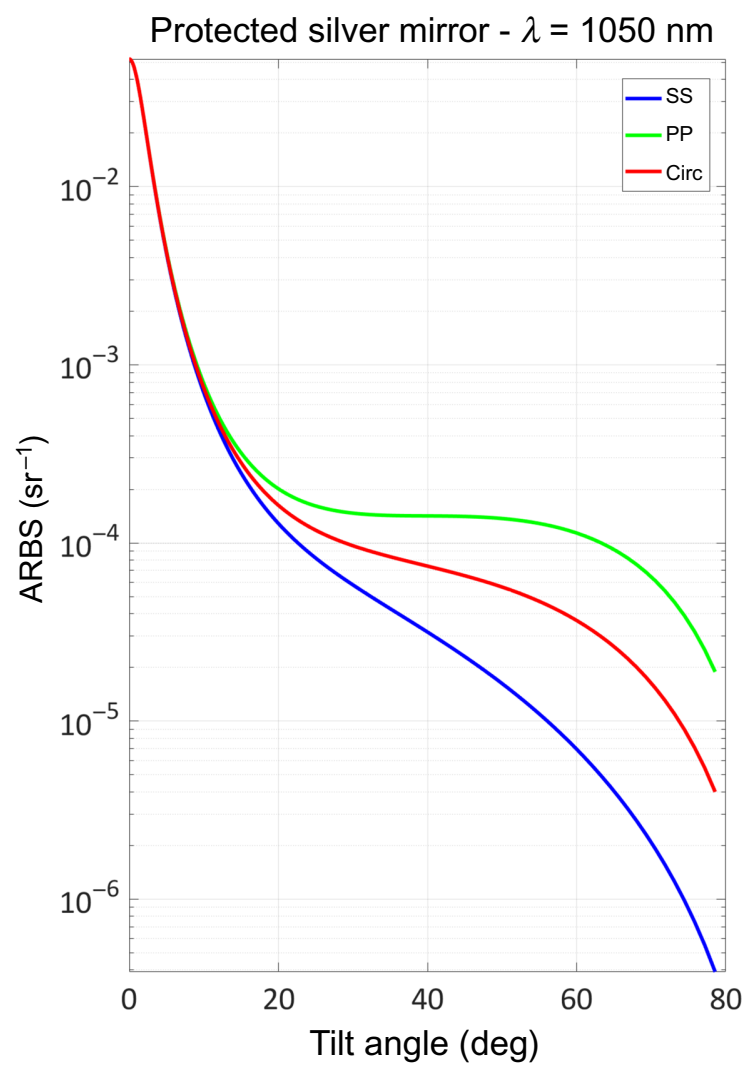

FIG. 13. Simulation of the angular dependence of the light back-scattered by a protected silver mirror (rms roughness $\approx 4$ $\mathrm{nm}$ ) for three states of polarization. 
the Nirvana receiver as follows:

$$
\begin{aligned}
V(\theta, \Delta)= & 2 \eta_{a}\left(\sqrt{T_{\mathrm{ref}, 1}^{\mathrm{TSR}} T_{\mathrm{sig}, 1}^{\mathrm{TSR}}}+\cos ^{2} \beta \sqrt{T_{\mathrm{ref}, 2}^{\mathrm{TSR}} T_{\mathrm{sig}, 2}^{\mathrm{TSR}}}\right) \\
& \times G S\left(f_{0}\right) P_{0} \sin 2 \alpha\left[\sqrt{R_{S}} e^{-(1 / 2)\left(2 \pi w_{f} \sin \theta / \lambda\right)^{2}}\right. \\
& +\sqrt{\operatorname{ARBS}(\theta) d \Omega}] e^{-(\pi \delta f \Delta / c)^{2}} \cos \left(k_{0} \Delta+\Psi\right),
\end{aligned}
$$

where, as previously, $\beta$ is the amplitude of the small rotation applied to the orientation of the linear polarizer, $\mathrm{LP}_{2}$ (polarization axis approximately horizontal), and $d \Omega$ is the solid angle corresponding to the divergence, $\delta \theta$, of the Gaussian illuminating beam, i.e.,

$$
d \Omega=\pi(\delta \theta)^{2}=\frac{\lambda^{2}}{\pi w_{s}^{2}} .
$$

The recording of $V(\theta, 0)$ thus gives access to the angular dependence of the back-reflectance, $\rho$, i.e., the ratio between the light power back-reflected and/or backscattered by the protected silver mirror and the illumination power. As highlighted by the results of theoretical modeling shown in Fig. 14 and using the same roughness data as

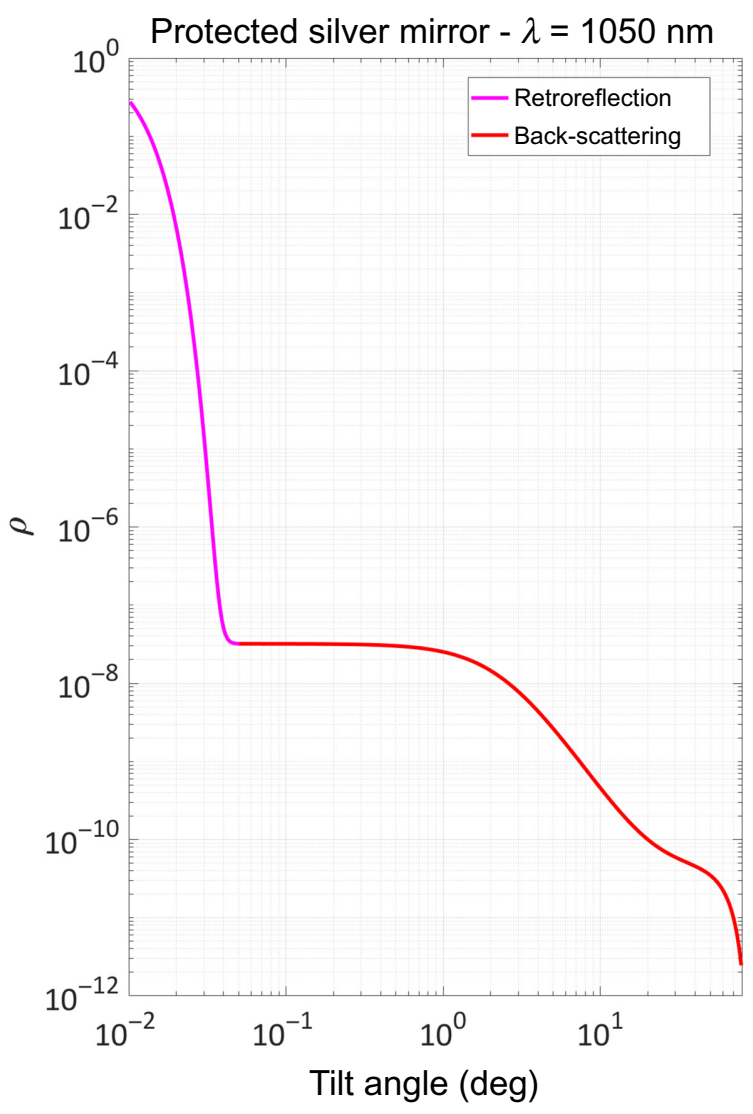

FIG. 14. Simulation of the angular dependence of the backreflectance, $\rho$, of a protected silver mirror (magenta curve, retroreflection; red curve, back-scattering). that in Fig. 13, there is a continuous transition between two regimes: the first at very low mirror tilt angles $\left(\theta \leqslant 0.04^{\circ}\right.$, retroreflection regime) and the second at greater angles $\left(\theta>0.04^{\circ}\right.$, back-scattering regime).

\section{Experimental results}

Figure 15(a) shows the time variation of the $V_{\text {ac }}$ signal experimentally recorded with the setup described in Sec. II and modified in accordance with Fig. 12, for different tilt angles of the protected silver mirror, while Fig. 15(b) presents the same data after frequency filtering (see Sec. III A). We note on these last curves a slight lateral displacement of the echo when the tilt angle increases; this is simply due to the presence of a residual offset between the rotation axis and the front face of the mirror.

Figure 16 shows the angular dependence of the backreflectance, $\rho$, derived from these interferometric measurements. First, note that the overall shape and range of variation of this experimental curve is comparable with that shown in Fig. 14. The relative accuracy of these backreflectance measurements is the same as that demonstrated in Sec. III B, i.e., about $20 \%$, while the accuracy of the definition of the tilt angle, $\theta$, is determined by the performance of the motorized rotation stage, i.e., a bidirectional repeatability of $\pm 0.1^{\circ}$ and a minimum incremental motion
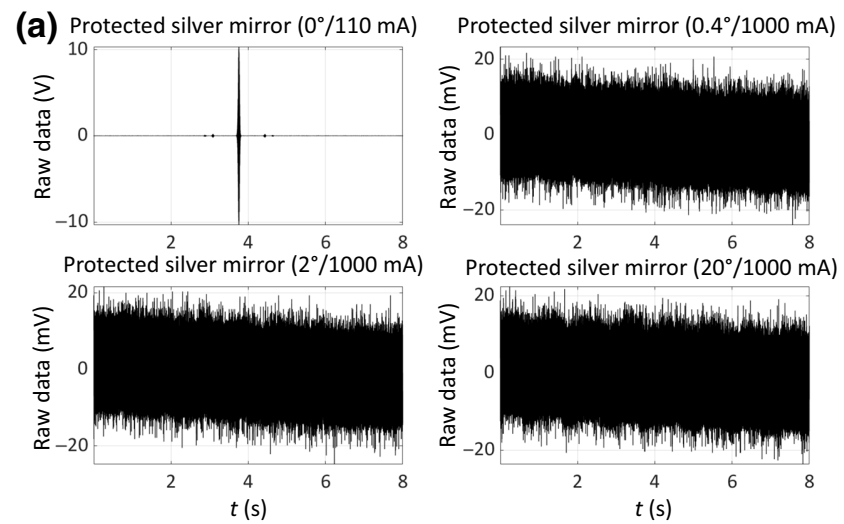

(b) Protected silver mirror $(0 \% 110 \mathrm{~mA})$

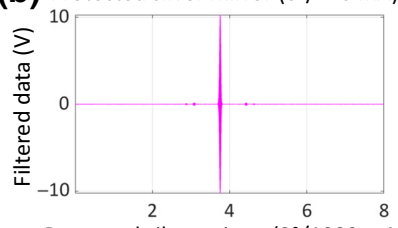

Protected silver mirror $\left(0.4^{\circ} / 1000 \mathrm{~mA}\right)$
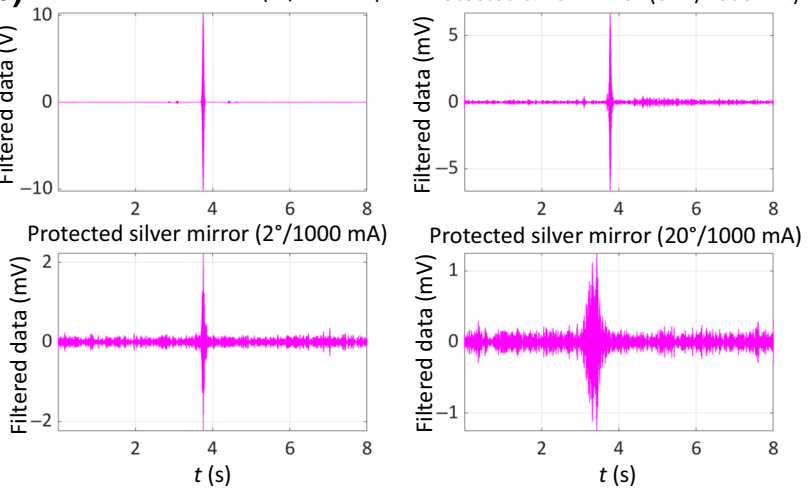

FIG. 15. Back-scattered signal, $V_{\text {ac }}$, measured from a protected silver mirror at different tilt angles: (a) raw data, (b) filtered data. 


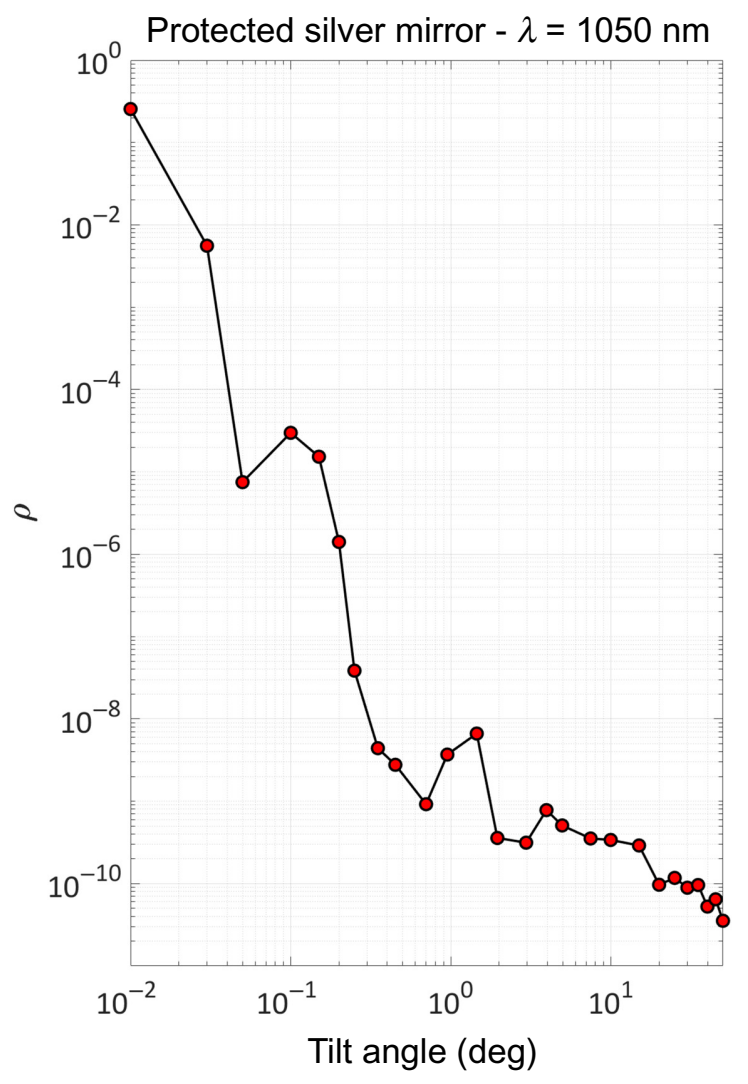

FIG. 16. Experimental measurement of the angular dependence of the back-reflectance, $\rho$, of a protected silver mirror (log-log units).

of $0.04^{\circ}$. These values are greater than the angular width of the speckle oscillations that modulate the amplitude of the back-scattering signal (typically $0.025^{\circ}$ ). This prevents any fine recording of these speckle oscillations using such a rotation stage and leads to undersampling of the angular variation of the back-reflectance, $\rho$. The presence of such a speckle effect is typical of any scatterometer that uses a laser beam as its illuminating source and is due to the very rapid variations of the roughness spectrum, $\gamma(\vec{v})$, with the spatial frequency, $\vec{v}$, even when averaged over the illuminated area and the source bandwidth. In Sec. IV $\mathrm{B}$, as highlighted, our theoretical modeling uses a polar mean, $\bar{\gamma}(v)$, that explains the lack of this speckle pattern in Figs. 13 and 14.

\section{CONCLUSION}

We present the measurement results of ultralow light power back-reflected or back-scattered by optical interfaces using balanced low-coherence interferometry. This method ensures easy identification of the scattering interface and allows the accurate measurement of backreflectance as low as a few $10^{-11}$. The implementation of this scheme for the measurement of light back-scattered by a tilted silver mirror is also demonstrated.
In the next few months, we intend to implement the following upgrades to this setup:

(a) the use of a linearly polarized version of the superluminescent diode (increasing the incident power by a factor of 2);

(b) an increase in the translation speed of the hollow retroreflector $(10 \mathrm{~mm} / \mathrm{s}$ instead of $0.5 \mathrm{~mm} / \mathrm{s})$, to shift the carrier frequency up to $20 \mathrm{kHz}$, which allows the Nirvana receiver to be operated in autobalanced mode (instead of simply balanced) and improves the rejection of source noise. However, this increase in translation speed needs the implementation of an alternative acquisition module, still with a 16-bit dynamic range, but with a higher sampling rate $(2 \mathrm{MS} / \mathrm{s})$;

(c) replacement of the Thorlabs PRMTZ8 motorized rotation stage by a Newport RGV100BL-S model with improved performance (bidirectional repeatability, \pm 0.15 mdeg; minimum incremental motion, $0.10 \mathrm{mdeg}$ ). This will allow the speckle pattern modulating the backreflectance signal to be accurately recorded and eliminated by averaging.

With all these upgrades, the sensitivity of the setup should be improved down to $10^{-13}$, which makes it possible to independently record the light scattered by the two faces of a glass window. This opens the door to the characterization of the scattering properties of various optical components, such as windows, coated or uncoated; all-dielectric and metallic mirrors; beam dumps; and optical assemblies.

\section{ACKNOWLEDGMENTS}

This work is part of the StrayLight Working Group for the Laser Instrument Group of the LISA Consortium. The authors would like to thank the AMIdex Talents Management Program, Aix Marseille University, and the French National Space Center (CNES) for their financial support.

[1] B. P. Abbott, et al., Observation of Gravitational Waves from a Binary Black Hole Merger, Phys. Rev. Lett. 116, 061102 (2016).

[2] J.-Y. Vinet, V. Brisson, and S. Braccini, Scattered light noise in gravitational wave interferometric detectors: Coherent effects, Phys. Rev. D 54, 1276 (1996).

[3] J.-Y. Vinet, V. Brisson, S. Braccini, I. Ferrante, L. Pinard, F. Bondu, and E. Tournié, Scattered light noise in gravitational wave interferometric detectors: A statistical approach, Phys. Rev. D 56, 6085 (1997).

[4] R. Takahashi, K. Arai, S. Kawamura, and M. R. Smith, Direct measurement of the scattered light effect on the sensitivity in TAMA300, Phys. Rev. D 70, 062003 (2004).

[5] F. Acernese, et al., Noise studies during the first Virgo science run data, Class. Quantum Grav. 25, 184003 (2008). 
[6] T. Accadia, et al., Noise from scattered light in Virgo's second science run data, Class. Quantum Grav. 27, 194011 (2010).

[7] A. Spector and G. Mueller, Back-reflection from a Cassegrain telescope for space-based interferometric gravitational-wave detectors, Class. Quantum Grav. 29, 205005 (2012).

[8] D. J. Ottaway, P. Fritschel, and S. J. Waldman, Impact of upconverted scattered light on advanced interferometric gravitational wave detectors, Opt. Express 20, 8329 (2012).

[9] B. Canuel, E. Genin, G. Vajente, and J. Marque, Displacement noise from back scattering and specular reflection of input optics in advanced gravitational wave detectors, Opt. Express 21, 10546 (2013).

[10] S. Zeidler, T. Akutsu, Y. Torii, E. Hirose, Y. Aso, and R. Flaminio, Calculation method for light scattering caused by multilayer coated mirrors in gravitational wave detectors, Opt. Express 25, 4741 (2017).

[11] F. Magaña-Sandoval, R. X. Adhikari, V. Frolov, J. Harms, J. Lee, S. Sankar, P. R. Saulson, and J. R. Smith, Largeangle scattered light measurements for quantum-noise filter cavity design studies, J. Opt. Soc. Am. A 29, 1722 (2012).

[12] C. Padilla, P. Fritschel, F. Magaña-Sandoval, E. Muniz, J. R. Smith, and L. Zhang, Low scatter and ultra-low reflectivity measured in a fused silica window, Appl. Opt. 53, 1315 (2014).

[13] S. Zeidler, T. Akutsu, Y. Torii, and Y. Aso, Measuring scattering light distributions on high-absorptive surfaces for stray-light reduction in gravitational-wave detectors, Opt. Express 27, 16890 (2019).

[14] V. Khodnevych, S. Di Pace, J.-Y. Vinet, N. Dinu-Jaeger, and M. Lintz, in International Conference on Space Optics-ICSO 2018, edited by Z. Sodnik, N. Karafolas and B. Cugny (Proc. SPIE, Bellingham, WA, USA, 2018), vol. 11180, p. 111807 T.

[15] V. Khodnevych, Ph.D. thesis, Université Côte d'Azur, 2020.

[16] A. Earle, H. Ward, A. Taylor, D. Robertson, and M. PerreurLloyd, in LISA Symposium XIII (2020).

[17] H. Lefèvre, The Fiber-Optic Gyroscope (Artech House, London, UK, 1993).

[18] R. Takada, A. Himeno, and K. Yukimatsu, Phase-noise and shot-noise limited operations of low coherence optical time domain reflectometry, Appl. Phys. Lett. 59, 2483 (1991).

[19] K. Kasaya, K. Yoshikuni, and H. Ishii, Measurements of a semiconductor waveguide using low-coherence interferometric reflectometer, IEEE Photonics Technol. Lett. 8, 251 (1996).

[20] D. Huang, E. A. Swanson, C. P. Lin, J. S. Schuman, W. G. Stinson, W. Chang, M. R. Hee, T. Flotte, K. Gregory, C. A. Puliafito, and J. G. Fujimoto, Optical coherence tomography, Science 254, 1178 (1991).

[21] B. Liu and M. E. Brezinski, Theoretical and practical considerations on detection performance of time domain, Fourier domain, and swept source optical coherence tomography, J. Biomed. Opt. 12, 044007 (2007).

[22] A. Gh. Podoleanu, Unbalanced versus balanced operation in an optical coherence tomography system, Appl. Opt. 39, 173 (2000).
[23] M. Lequime and J. Lumeau, Accurate determination of the optical performances of antireflective coatings by low coherence reflectometry, Appl. Opt. 46, 5635 (2007).

[24] P. C. D. Hobbs, Ultrasensitive laser measurements without tears, Appl. Opt. 36, 903 (1997).

[25] M. Lequime, S. Liukaityte, M. Zerrad, and C. Amra, Ultrawide-range measurements of thin-film filter optical density over the visible and near-infrared spectrum, Opt. Express 23, 26863 (2015).

[26] M. Lequime, M. Zerrad, and C. Amra, Breakthrough spectrophotometric instrument for the ultra-fine characterization of the spectral transmittance of thin-film optical filters, Opt. Express 26, 34236 (2018).

[27] M. Fouchier, M. Zerrad, M. Lequime, and C. Amra, Widerange wavelength and angle resolved light scattering measurement apparatus, Opt. Lett. 45, 2506 (2020).

[28] C. Amra, M. Lequime, and M. Zerrad, Electromagnetic Optics of Thin-Film Coatings-Light Scattering, Giant Field Enhancement, and Planar Microcavities (Cambridge University Press, Cambridge, UK, 2021).

[29] M. Lequime and C. Amra, De l'Optique Électromagnétique à l'Interférométrie - Concepts et Illustrations (EdP Sciences, Paris, France, 2013), Chapter 19.

[30] https://shop.schott.com/advanced_optics/en/Optical-Glass/ SCHOTT-N-BK7/c/optical-glass/glass-SCHOTT\%20N-BK $7 \% \mathrm{C} 2 \% \mathrm{AE}$.

[31] https://www.oharacorp.com/pdf/datasheets/S-LAH66-2020 -06.pdf.

[32] T. Pikalek, T. Fort, and Z. Buchta, Detection techniques in low-coherence interferometry and their impact on overall measurement accuracy, Appl. Opt. 53, 8463 (2014).

[33] L. Xin, Z. Yang, J. Dou, Z. Liu, Z. Gao, and X. Zhang, Hilbert transform-based envelope substitution method for non-uniform sampling signal correction in white-light interferometry, OSA Contin. 3, 824 (2020).

[34] J. C. Stover, Optical Scattering: Measurement and Analysis (SPIE Press Book, Bellingham, WA, USA, 2012), 3rd ed.

[35] A. von Finck, et al., International round-robin experiment for angle-resolved light scattering measurement, Appl. Opt. 58, 6638 (2019).

[36] J. Degallaix, C. Michel, B. Sassolas, A. Allocca, G. Cagnoli, L. Balzarini, V. Dolique, R. Flaminio, D. Forest, M. Granata, B. Lagrange, N. Straniero, J. Teillon, and L. Pinard, Large and extremely low loss: The unique challenges of gravitational wave mirrors, J. Opt. Soc. Am. A 36, C85 (2019).

[37] P. J. Jobst, O. Stenzel, M. Schurmann, N. Modsching, S. Yulin, S. Wilbrandt, D. Gabler, N. Kaiser, and A. Tunnermann, Optical properties of unprotected and protected sputtered silver films: Surface morphology vs. UV/VIS reflectance, Adv. Opt. Technol. 3, 91 (2014).

[38] J. M. Elson, J. P. Rahn, and J. M. Bennett, Relationship of the total integrated scattering from multilayer-coated optics to angle of incidence, polarization, correlation length, and roughness cross-correlation properties, Appl. Opt. 22, 3207 (1983). 